\title{
A Demo of a Dynamic Facial UI for Digital Artists
}

\author{
Pedro Bastos ${ }^{1}$, Xenxo Alvarez ${ }^{2}$, and Veronica Orvalho ${ }^{2}$ \\ ${ }^{1}$ Instituto de Telecomunicações, Faculdade de Engenharia da Universidade do Porto, \\ Rua Dr. Roberto Frias, s/n 4200-465 Porto Portugal \\ ${ }^{2}$ Instituto de Telecomunicações, Faculdade de Ciências da Universidade do Porto, \\ Rua do Campo Alegre, s/n, 4169-007 Porto Portugal
}

\begin{abstract}
Character facial animation is difficult because the face of a character assumes many complex expressions. To achieve convincing visual results for animation, 3D digital artists need to prepare their characters with sophisticated control structures. One of the most important techniques to achieve good facial animation is to use facial control interfaces, also called facial user interfaces, or facial UI's. But facial UI's are usually dull and often confusing, with limited user interaction and no flexibility. We developed a concept and a working prototype of a dynamic facial UI inside the Blender [1] open-source software to allow their large community of digital artists to better control and organize the facial animation of a character. Our interactive system is running stable in the latest version of Blender and we started to build a full-face dynamic UI to show its interactive potential in a character's face.
\end{abstract}

Keywords: Accessibility and Usability, Novel User Interfaces and Interaction Techniques, Adaptive Interfaces, Dynamic Interfaces, Facial Interfaces, Facial Rigging and Animation, Digital Artists.

\section{Introduction}

Facial UI's [2] are GUI's (graphical user interfaces) with panels and controls that are used to facilitate the animation of a character's face. Facial UI's are usually placed side-by-side to the face of a 3D character in order for the animator to have easy access to its controls. The panels of a facial UI have a fixed position in space and generally each panel has a controller centered in the panel. The 2D spatial transformation of a control in a given panel affects the transformation of a corresponding locator in the skeleton of the character's mesh. The controls in the facial UI allow manipulating the character rig structure (the skeleton's bones) which in turn deforms the mesh.

The number of panels in the facial UI usually corresponds to the number of facial regions that need to be animated. The controls are grouped based on the anatomical areas of the face. There are references to help the digital artist build the animation controls for the face, such as the Facial Action Coding System [3].

The main benefit of a facial UI is the fact that it facilitates the understanding of the facial controls a character needs to have in order to simulate facial expressions [4] accurately. A facial UI enables the digital artist to interact with 2D controls that in fact report to $3 \mathrm{D}$ controls which are rigged to the mesh of the face of the character.

In essence, facial UI's prevent the visual confusion of the usual controls laid on top of the skin of the character. They also allow the user to have a better understanding of 
how each control is used, because the controls are constrained to the UI's panels. This prevents digital artists from doing unexpected manipulations of the controls which could result in awkward skin deformations and inaccurate facial animation.

But the common facial UI's also have considerable limitations. For instance, they occupy fixed positions on space. Also, the digital artist responsible for doing the UI generally places it side-by-side to the face of the character and the animator cannot change this. Also, facial UI's offer pre-established layouts with no flexibility for reorganization and re-dimensioning. This constrains and disorients the animator.

In general, a facial UI is a very complete system but is not straight forward enough nor it is the most user-friendly method, because the interactive abilities it offers are insufficient when compared to the flexibility of controls placed in the skin.

\section{Our Solution: A Dynamic Facial UI}

We developed a concept and a prototype of a dynamic facial UI in the Blender [1] software to help the large community of Blender digital artists do better animations for their characters faces. Our method improves the interaction of digital artists with facial UI's. It is an innovative technical solution for the 3D digital artist based on an extension of the features found in common facial UI's.

We already have a working prototype with very interesting features, such as the ability for the animator to easily relocate and re-dimension the panels in a facial UI. The animator can customize the limits and rearrange the layout of the facial UI, gaining more control over the animation of the several facial regions. It is possible to re-position the entire UI and/or a specific panel, transforming it to fit in the facial region as desired. The option to configure each panel provides more $2 \mathrm{D}$ space for manipulating the controls and a much better visualization and interaction.

Our prototype is able to join the abilities of the controls placed over the skin of the character to the abilities found in common facial UI's. With our prototype the digital artist can animate the face using the best of both worlds: having the flexibility of the controls placed directly in the skin and the benefits of using a facial UI.

Our concept has rendered us an article in the BlenderArt magazine. But we haven't yet had the opportunity to show it as a running demo. We would like to show it to an audience for the first time in the Interact2011 conference, with new improved functionalities and with the prototype applied to the several regions of the face.

We are also working to fully automate the process and make it compatible with any facial style the digital artist may need to animate. We attach a video to the submission of this document showing our results at the current development stage.

\section{References}

1. Blender Foundation, http://www.blender.org

2. Osipa, J.: Stop Staring: Facial Modeling and Animation Done Right. Sybex, Wiley Pub., Indianapolis, Indiana, USA (2010)

3. Ekman, P., Friesen, W.: The Facial Action Coding System. Research Nexus. Network Information Research Corporation, Salt Lake City, USA (2002)

4. Faigin, G.: The Artist's Complete Guide to Facial Expressions. Watson-Guptill Pub., New York (1990) 